\title{
Symmetry energy and density
}

\author{
Wolfgang TRAUTMANN* \\ GSI Helmholtzzentrum Gmbh, D-64291 Darmstadt, Germany \\ E-mail: w.trautmannegsi.de
}

\section{Mircea Dan COZMA}

IFIN-HH, Reactorului 30, 077125 Măgurele-Bucharest, Romania

E-mail: dan.cozma@theory.nipne.ro

\section{Paolo RUSSOTTO}

INFN-Sezione di Catania, I-95123 Catania, Italy

E-mail: russotto@lns.infn.it

\begin{abstract}
The nuclear equation-of-state is a topic of highest current interest in nuclear structure and reactions as well as in astrophysics. In particular, the equation-of-state of asymmetric matter and the symmetry energy representing the difference between the energy densities of neutron matter and of symmetric nuclear matter are not sufficiently well constrained at present. The density dependence of the symmetry energy is conventionally expressed in the form of the slope parameter $L$ describing the derivative with respect to density of the symmetry energy at saturation. Results deduced from nuclear structure and heavy-ion reaction data are distributed around a mean value $L=60 \mathrm{MeV}$.

Recent studies have more thoroughly investigated the density range that a particular observable is predominantly sensitive to. Two thirds of the saturation density is a value typical for the information contained in nuclear-structure data. Higher values exceeding saturation have been shown to be probed with meson production and collective flows at incident energies in the range of up to about $1 \mathrm{GeV} /$ nucleon.

From the measurement of the elliptic-flow ratio of neutrons with respect to light charged particles in recent experiments at the GSI laboratory, a new more stringent constraint for the symmetry energy at suprasaturation density has been deduced. It confirms, with a considerably smaller uncertainty, the moderately soft to linear density dependence of the symmetry energy previously deduced from the FOPI-LAND data. Future opportunities offered by FAIR will be discussed.
\end{abstract}

54th International Winter Meeting on Nuclear Physics

25-29 January 2016

Bormio, Italy

${ }^{*}$ Speaker. 


\section{Introduction}

In a recent paper, $\mathrm{Li}$ and Han have documented that the many results obtained for the nuclear symmetry energy from terrestrial nuclear experiments and astrophysical observations are amazingly compatible, even though individual results scatter within certain margins and are partly affected with considerable errors [1]. The average values deduced by the authors for the symmetry energy at saturation density and for the slope parameter describing its density dependence are $E_{\text {sym }}\left(\rho_{0}\right)=31.6 \mathrm{MeV}$ and $L=58.9 \mathrm{MeV}$, respectively. The parameter $L$, defined as

$$
L=\left.3 \rho_{0} \frac{\partial E_{\text {sym }}}{\partial \rho}\right|_{\rho=\rho_{0}},
$$

is proportional to the slope of the symmetry energy at saturation density $\rho_{0}$ (see, e.g., Ref. [2]). The authors quote also error margins representative for the variation of the individual results as $\Delta E_{\text {sym }}\left(\rho_{0}\right)=2.7 \mathrm{MeV}$ and $\Delta L=16 \mathrm{MeV}$ and conclude that $L$ has a value about twice as large as $E_{\text {sym }}\left(\rho_{0}\right)$. A very similar conclusion can be drawn from the compilation of Lattimer and Steiner [3] adapted from the earlier work of Lattimer and Lim [4].

The nuclear symmetry energy appears in many aspects of nuclear structure and reactions and determines very basic properties of neutron stars as, e.g., their radii $[2,5]$. This implies that many different sources of information exist, in the laboratory and in the cosmos, that provide information on the equation of state (EoS) of asymmetric nuclear matter. Comparisons of the mentioned kind are possible because the forces identified as best describing a particular observation can be used in many-body calculations for generating results for the two quantities characterizing the asymmetricmatter EoS at saturation density. It does not require that the measurement or observation has actually tested the EoS at this density. The result may represent an extrapolation.

The predictions of microscopic models for the nuclear symmetry energy, obtained with realistic or phenomenological forces, appear to coincide at densities close to $\rho=0.1 \mathrm{fm}^{-3}$, i.e. at approximately two thirds of the saturation density [6]. It reflects the fact that the average density of atomic nuclei is below saturation and that the presence of the surface influences the nuclear properties that are chosen as constraints. The awareness that each observable carrying information on the nuclear EoS is connected to its proper range of density has to complement the interpretation of existing results.

Rather precise values for the symmetry energy and for the density to which it applies have recently been presented by Brown [7] and Zhang and Chen [8]. They are shown in Fig. 1 together with the low-density behavior of the symmetry energy obtained from heavy-ion collisions [9] and from the analysis of isobaric analog states [10] as reported in Ref. [11]. In the study of Brown, a set of selected Skyrme forces is used whose parameters are fitted to properties of doubly magic nuclei. By using particular values for the neutron skin of ${ }^{208} \mathrm{~Pb}$ nuclei within a given range as additional constraints, new sets of of these forces with slightly adjusted parameters are obtained. It is found that all predictions coincide at a density $\rho=0.1 \mathrm{fm}^{-3}$, independent of the choice made for the thickness of the neutron skin, but that the slopes at this density depend on this choice. Only a precise knowledge of the neutron skin of ${ }^{208} \mathrm{~Pb}$ will permit the extrapolation to the saturation point. It underlines the importance of this quantity determined by the balance of pressures felt by neutrons in the neutron-enriched interior of the ${ }^{208} \mathrm{~Pb}$ nucleus at approximately saturation density and in the low-density neutron-rich surface $[12,13,14]$. 
Sensitivities to higher densities can be expected from observables related to the early phases of heavy-ion collisions at sufficiently high energies. Calculations predict that densities up to three times the saturation value are reached for short times $(\approx 20 \mathrm{fm} / c)$ in the central zone of heavy-ion collisions with energies of up to $\approx 1 \mathrm{GeV} /$ nucleon [15]. Twice the saturation value may already be reached at the moderate energy of $400 \mathrm{MeV} /$ nucleon at which the reaction dynamics is still largely determined by the nuclear mean field. The resulting pressure produces a collective outward motion of the compressed material whose strength will be influenced by the symmetry energy in asymmetric systems [16]. A measurement differentiating between the collective flows of neutrons and protons can thus be expected to provide information on the high-density symmetry energy [17].

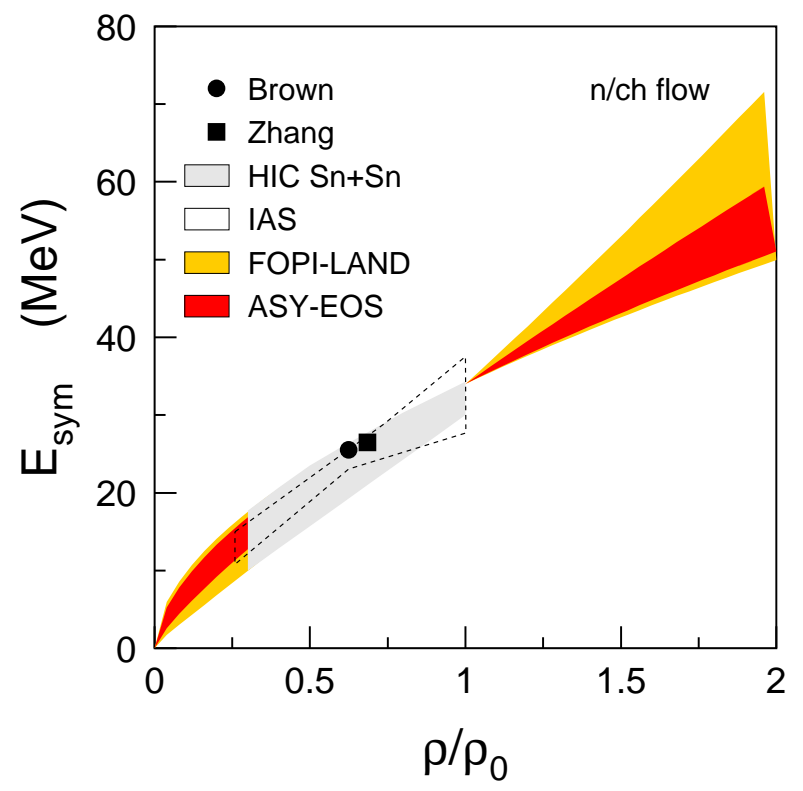

Figure 1: (Color online) Constraints deduced for the density dependence of the symmetry energy from the ASY-EOS data [28] in comparison with the FOPI-LAND result of Ref. [18] as a function of the reduced density $\rho / \rho_{0}$. The low-density results of Refs. [7, 8, 9, 10] as reported in Ref. [11] are given by the symbols, the grey area (HIC), and the dashed contour (IAS). For clarity, the FOPI-LAND and ASY-EOS results are not displayed in the interval $0.3<\rho / \rho_{0}<1.0$ (from Ref. [28]; Copyright (2016) by the American Physical Society).

The ratio of elliptic flow strengths observed for neutrons and light charged particles has been proposed as an observable sensitive to the EoS of asymmetric matter [18]. From a reanalysis of the earlier FOPI-LAND data $[19,20]$ and the comparison with calculations performed with the UrQMD transport model [21, 22, 23] a moderately soft to linear symmetry term, characterized by a coefficient $\gamma=0.9 \pm 0.4$ for the power-law parametrization of the density dependence of the potential part of the symmetry energy was obtained [18, 24, 25, 26, 27]. It is represented by the yellow band in Fig. 1. Motivated by this finding, an attempt has been made to improve the accuracy with a new experiment that was conducted at the GSI laboratory in 2011 (ASY-EOS experiment S394). The new result, reported in Ref. [28], is represented by the red band in the figure. Both results are displayed over the range of densities up to twice saturation. It will be shown in this talk that the sensitivity of this particular observable reaches even beyond that point. 


\section{The ASY-EOS experiment}

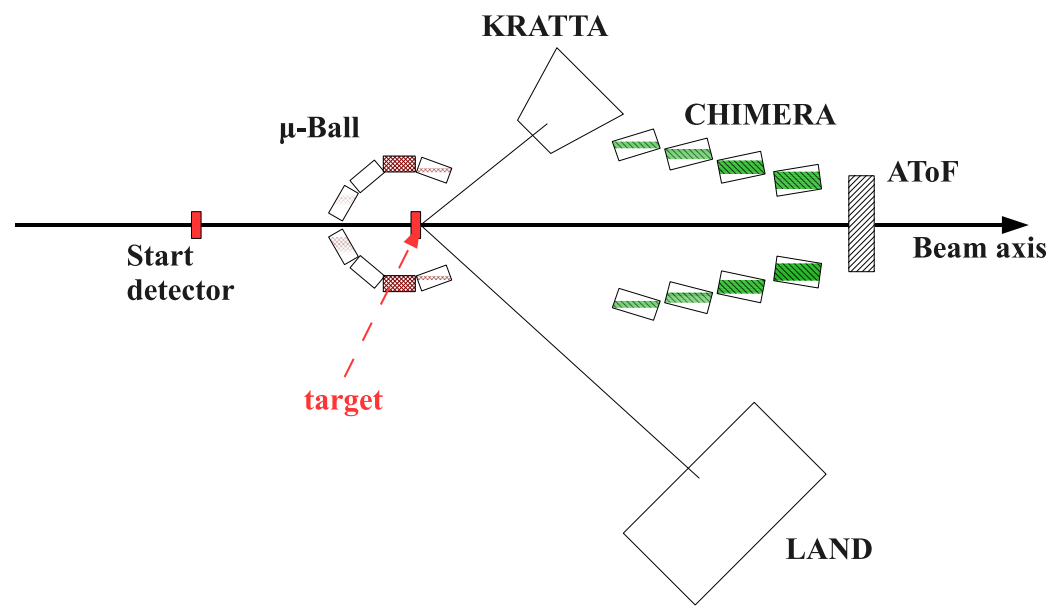

Figure 2: (Color online) Schematic view of the experimental setup used in the ASY-EOS experiment S394 at the GSI laboratory showing the six main detector systems and their positions relative to the beam direction. The dimensions of the symbols and the distances are not to scale (from Ref. [29]).

The experimental setup of the ASY-EOS experiment at the GSI laboratory followed the scheme developed for FOPI-LAND by using the Large Area Neutron Detector (LAND [30]) as the main instrument for neutron and charged particle detection (Fig. 2). Several detection systems with azimuthal symmetry with respect to the beam axis served in determining the orientation of the reaction plane. Upstream from the target, a thin plastic scintillator foil viewed by two photomultipliers was used to record the projectile arrival times and to serve as a start detector for the time-of-flight measurement. LAND was positioned at a laboratory angle close to $45^{\circ}$ with respect to the beam direction. A veto wall of thin plastic scintillators in front of LAND was used for discriminating between neutrons and charged particles. This configuration permitted the measurement of directed and elliptic flows of neutrons and charged particles near mid-rapidity within the same angular acceptance. Opposite of LAND, covering a comparable range of polar angles, the Kraków Triple Telescope Array (KRATTA [31]) was installed to permit flow measurements of identified charged particles under the same experimental conditions.

For the event characterization and for measuring the orientation of the reaction plane, three detection systems had been installed. The ALADIN Time-of-Flight (AToF) Wall [32] was used to detect charged particles and fragments in forward direction at polar angles up to $\theta_{\text {lab }} \leq 7^{\circ}$. Its capability of identifying large fragments and of characterizing events with a measurement of $Z_{\text {bound }}$ [32] permitted the sorting of events according to impact parameter. Four double rings of the CHIMERA multidetector [33, 34] carrying together $352 \mathrm{CsI}(\mathrm{Tl})$ scintillators in forward direction and four rings with 50 thin $\mathrm{CsI}(\mathrm{Tl})$ elements of the Washington University Microball array [35] surrounding the target provided sufficient coverage and granularity for determining the orientation of the reaction plane from the measured azimuthal particle distributions. A detailed description of the experiment is available in Ref. [28]. 


\section{Experimental results}

As in the FOPI-LAND experiment, the reaction ${ }^{197} \mathrm{Au}+{ }^{197} \mathrm{Au}$ at $400 \mathrm{MeV} /$ nucleon was studied. The elliptic flows of neutrons and light charged particles were determined from the azimuthal distributions of these particles with respect to the reaction plane reconstructed from the distributions of particles and fragments recorded with the three arrays AToF, CHIMERA, and Microball. Methods developed and described in Refs. [36, 37] for correcting the finite dispersion of the reaction plane were applied. The coefficients $v_{1}$ and $v_{2}$ representing the strengths of directed and elliptic flows, respectively, were deduced from fits with the Fourier expansion

$$
f(\Delta \phi) \propto 1+2 v_{1} \cos (\Delta \phi)+2 v_{2} \cos (2 \Delta \phi) .
$$

Here $\Delta \phi$ represents the azimuthal angle of the momentum vector of an emitted particle with respect to the angle representing the azimuthal orientation of the reaction plane. Constraints for the symmetry energy were determined by comparing the ratios of the elliptic flows of neutrons and charged particles (ch), $v_{2}^{n} / v_{2}^{c h}$, with the corresponding UrQMD predictions for soft and stiff assumptions.

The UrQMD model was originally developed to study particle production at high energy [38]. By introducing a nuclear mean field with momentum dependent forces, it has been adapted to the study of intermediate energy heavy-ion collisions [39]. The updated Pauli-blocking scheme, introduced to provide a more precise description of experimental observables at lower energies, is described in Ref. [40]. The chosen isoscalar EoS is soft and different options for the dependence on isospin asymmetry were implemented. Two of them are used here, expressed as a power-law dependence of the potential part of the symmetry energy on the nuclear density $\rho$ according to

$$
E_{\mathrm{sym}}=E_{\mathrm{sym}}^{\mathrm{pot}}+E_{\mathrm{sym}}^{\mathrm{kin}}=22 \operatorname{MeV}\left(\rho / \rho_{0}\right)^{\gamma}+12 \operatorname{MeV}\left(\rho / \rho_{0}\right)^{2 / 3}
$$

with $\gamma=0.5$ and $\gamma=1.5$ corresponding to a soft and a stiff density dependence, respectively.

The predictions obtained with these assumptions for the measured flow ratio are shown in Fig. 3 together with the experimental result. The histogram represents the linear interpolation between the predictions giving a best fit of the flow ratios presented here as a function of the transverse momentum $p_{t}$. The corresponding power-law coefficient is $\gamma=0.75 \pm 0.10$. In comparison with FOPI-LAND, the statistical accuracy $\Delta \gamma=0.10$ represents a strong improvement by more than a factor of two. A systematic uncertainty arose from occasional malfunctions of the electronic circuits for the time measurement with LAND. It prohibits extending the analysis into the weakly polulated region with $p_{t}>0.7 \mathrm{MeV} / c$. It is of considerably less importance for the flow ratio constructed after integrating the measured yields over the full acceptance of LAND in the $p_{t}$-vs-rapidity plane. Only a minor uncertainty remains that is related to the lower energy threshold of neutron detection. The necessary corrections and the methods used for estimating the resulting errors are described in detail in Ref. [28]. They include, e.g., a small correction caused by the possible misinterpretation of neutrons as charged particles and vice versa, caused by reactions of neutrons in the veto wall and by the narrow gaps between the veto-wall paddles.

With all corrections and errors included, the acceptance-integrated elliptic-flow ratio leads to a power-law coefficient $\gamma=0.72 \pm 0.19$. This is the result displayed in Fig. 1 as a function of the reduced density $\rho / \rho_{0}$. The new result confirms the former and has a considerably smaller 


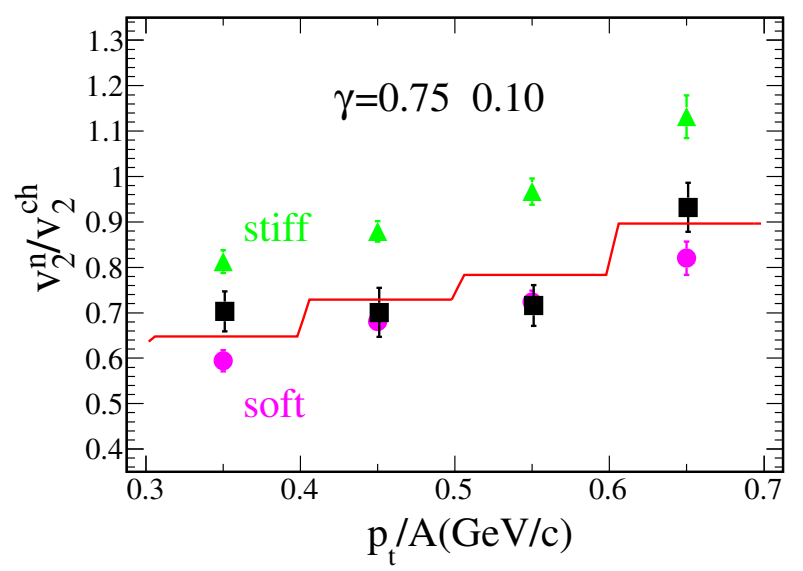

Figure 3: (Color online) Elliptic flow ratio of neutrons over all charged particles for central $(b<7.5 \mathrm{fm})$ collisions of ${ }^{197} \mathrm{Au}+{ }^{197} \mathrm{Au}$ at $400 \mathrm{MeV} /$ nucleon as a function of the transverse momentum/nucleon $p_{t} / A$. The black squares represent the experimental data, the green triangles and purple circles represent the UrQMD predictions for stiff $(\gamma=1.5)$ and soft $(\gamma=0.5)$ power-law exponents of the potential term, respectively. The solid line is the result of a linear interpolation between the predictions, weighted according to the experimental errors of the included four bins in $p_{t} / A$, and leading to the indicated $\gamma=0.75 \pm 0.10$ (from Ref. [28]; Copyright (2016) by the American Physical Society).

uncertainty. It is also worth noting that the present parametrization is compatible with the lowdensity behavior of the symmetry energy from Refs. $[7,8,9,10]$ that are included in the figure. The corresponding slope parameter describing the variation of the symmetry energy with density at saturation is $L=72 \pm 13 \mathrm{MeV}$. The sharp value $E_{\text {sym }}\left(\rho_{0}\right)=34 \mathrm{MeV}$ is a consequence of the chosen parametrization (Eq. 3.2). Using values lower than the default $E_{\mathrm{sym}}^{\mathrm{pot}}\left(\rho_{0}\right)=22 \mathrm{MeV}$, as occasionally done in other UrQMD studies [41], will lower the result for $L$. Performing the present UrQMD analysis with $E_{\mathrm{sym}}^{\text {pot }}\left(\rho_{0}\right)=19 \mathrm{MeV}$, corresponding to $E_{\mathrm{sym}}\left(\rho_{0}\right)=31 \mathrm{MeV}$, yields a powerlaw coefficient $\gamma=0.68 \pm 0.19$ and a slope parameter $L=63 \pm 11 \mathrm{MeV}$. The observed changes remain both within the error margins of these quantities. However, the precise results of Brown [7] and Zhang and Chen [8] are not equally met with this alternative parametrization of the symmetry energy.

\section{Sensitivity to density}

The sensitivity to density was explored in a study using the Tübingen version [42] of the QMD model (TüQMD). The underlying idea consisted in performing transport calculations for the present reaction with two parametrizations of the symmetry energy that were chosen to be different for a selected range of density and identical elsewhere. The magnitude of the obtained difference between the two predictions for the elliptic flow ratio is considered a measure of the sensitivity to the selected density region.

In the momentum-dependent one-body potential introduced by Das et al. [43], the stiffness of the symmetry energy is controlled with a parameter $x$ and choices ranging from soft $(x=+1)$ up to rather stiff $(x=-2)$ density dependences are commonly selected in model studies. For the present case, a mildly stiff $(x=-1)$ and a soft $(x=+1)$ parametrization were chosen for the density range 


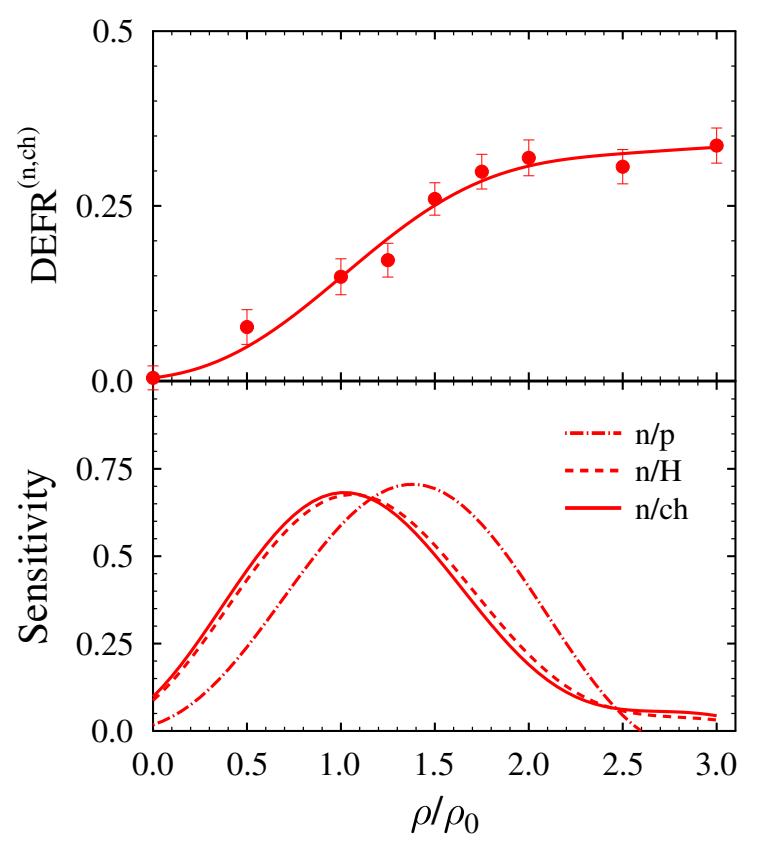

Figure 4: Density dependence of the difference of the elliptic flow ratio (DEFR) of neutrons over charged particles, defined by Eq. 4.1, for ${ }^{197} \mathrm{Au}+{ }^{197} \mathrm{Au}$ collisions at $400 \mathrm{MeV} /$ nucleon obtained with the TüQMD transport model [42] and the FOPI-LAND acceptance filter (top) and the corresponding sensitivity density (bottom panel, solid line) together with sensitivity densities obtained from elliptic-flow ratios of neutrons over all hydrogen isotopes (dashed) and neutrons over protons (dash-dotted; from Ref. [28]; Copyright (2016) by the American Physical Society).

with different symmetry energy while the nearly linear case with $x=0$ was chosen for the common part.

To quantify the results, a quantity DEFR (Difference of Elliptic-Flow Ratio) was defined as

$$
\operatorname{DEFR}^{(n, Y)}(\rho)=\frac{v_{2}^{n}}{v_{2}^{Y}}(x=-1, \rho)-\frac{v_{2}^{n}}{v_{2}^{Y}}(x=1, \rho) .
$$

It represents the differences of the elliptic flow ratios calculated with the TüQMD transport model for two different density dependencies of the symmetry energy. Here $Y$ indicates a particle or a group of particle species and $x$ the stiffness that is used in the calculations at densities smaller than the argument $\rho$. The difference of the $x= \pm 1$ potentials is thus effectively only tested at densities up to the particular $\rho$, the argument of DEFR. This choice leads to $\operatorname{DEFR}^{(n, Y)}(0)=0$ and to the full stiff-soft splitting for large values of the argument $\rho$. The slope of DEFR at intermediate densities is a measure of the impact on elliptic flow observables of that particular region of density.

In the upper panel of Fig. 4, the density dependence of $\operatorname{DEFR}^{(n, Y)}$ for the choice $Y=$ all charged particles is presented. It is seen that DEFR increases monotonically up to density values in the neighborhood of $2.5 \rho_{0}$, close to the maximum density probed by nucleons in heavy-ion collisions at $400 \mathrm{MeV} /$ nucleon incident energy. The distribution of the sensitivity as a function of density is obtained by forming the derivative of DEFR with respect to density. It is presented in the lower panel of Fig. 4 for three choices of $Y$, only protons (n/p), sum of all hydrogen isotopes $(\mathrm{n} / \mathrm{H})$, and all charged particles (n/ch). 
The figure shows that the sensitivity achieved with the elliptic-flow ratio of neutrons over charged particles, the case studied here, reaches its maximum close to saturation density and extends beyond twice that value. It is compatible with the conclusions reached by Le Fèvre et al. in their study of the symmetric matter EoS, based on FOPI elliptic-flow data and calculations with the Isospin Quantum Molecular Dynamics transport model [44]. For ${ }^{197} \mathrm{Au}+{ }^{197} \mathrm{Au}$ collisions at 400 $\mathrm{MeV} /$ nucleon, the broad maximum of the force-weighted density defined by the authors is spread over the density range $0.8<\rho / \rho_{0}<1.6$.

The sensitivity of the neutron-vs-proton flow ratio has its maximum in the 1.4 to $1.5 \rho_{0}$ region, i.e. at significantly higher densities than with light complex particles being included (Fig. 4). This observation contains an important potential for future experiments. With efficient isotope separation, flow measurements may give access to the curvature of the symmetry energy at saturation, in addition to the slope. It is, therefore, of extreme importance for future experiments to be able to extract a clean separate proton signal. Theoretical models to be used in the analysis should permit the independent adjustment of the slope and curvature parameters of the symmetry-energy term. With improvements in these directions and higher beam energies, extracted constraints for the EoS of asymmetric matter can be expected to reach into the $2 \rho_{0}$ regime.

\section{Conclusion and Outlook}

The sensitivity study shows that suprasaturation densities are effectively probed with the elliptic flow ratio of neutrons with respect to charged particles. Because the interpretation of the FOPI pion ratios [45] is not yet conclusive (see, e.g., Refs. [46, 47, 48]), this observable is presently unique as a terrestrial source of information for the EoS of asymmetric matter at high densities.

The value $\gamma=0.72 \pm 0.19$ obtained for the power-law coefficient of the potential part in the UrQMD parametrization of the symmetry energy and the slope parameter $L=72 \pm 13 \mathrm{MeV}$ are equivalent to a symmetry pressure $p_{0}=\rho_{0} L / 3=3.8 \pm 0.7 \mathrm{MeVfm}^{-3}$. The latter may be used to estimate the pressure in neutron-star matter at saturation density. For an assumed asymmetry $\delta=\left(\rho_{n}-\rho_{p}\right) / \rho=0.9$ in that part of the star, it amounts to $3.4 \mathrm{MeVfm}^{-3}$ (Ref. [28]), a value that compares well with the pressure obtained by Steiner et al. [49] from neutron-star observations.

Regarding the modeling of nuclear reactions, it will be important to improve the description of the nuclear interaction in transport models, to reduce the parameter ranges also in the isoscalar sector, to improve the algorithms used for clusterization, as well as going beyond the mean-field picture, including short-range correlations. The latter have recently been investigated in nuclei [50, 51] and their consequences for transport descriptions of intermediate-energy heavy-ion reactions need to be investigated. Moreover, it will be quite important to compare the experimental data with the predictions of several transport models, of both Boltzmann-Vlasov and molecular-dynamics type [52], in order to pursue the work towards a model-independent constraint of the high-density symmetry energy initiated in Refs. [27, 42].

The presented experimental results and the theoretical study of the density range that has been probed, provide a strong encouragement for continuing flow measurements of the present kind with improved detection systems. Preliminary model studies indicate that the sensitivity to the stiffness of the symmetry energy is still significant at incident energies as high as $800 \mathrm{MeV}$ or even $1 \mathrm{GeV} /$ nucleon. The density study suggests that the curvature parameter $K_{\text {sym }}$ can be 
addressed experimentally if higher precision and elemental and isotopic resolution for charged particles can be achieved. Future experiments will, therefore, benefit from the improved capabilities of the NeuLAND detector [53] presently constructed as part of the $R^{3} B$ setup for experiments at FAIR. Continuing experimental and theoretical activities can thus be expected to drive the range of densities that can be probed in the laboratory up to twice saturation.

The results presented in this talk have been obtained within the ASY-EOS Collaboration. See Ref. [28] for the complete list of authors.

\section{References}

[1] Bao-An Li and Xiao Han, Phys. Lett. B 727 (2013) 276.

[2] for a review, see Bao-An Li, Lie-Wen Chen, Che Ming Ko, Phys. Rep. 464 (2008) 113.

[3] J. M. Lattimer and A. W. Steiner, Eur. Phys. J. A 50 (2014) 40.

[4] J. M. Lattimer and Y. Lim, Astrophys. J. 771 (2013) 51.

[5] Topical issue on nuclear symmetry energy, edited by Bao-An Li, Àngels Ramos), Giuseppe Verde, Isaac Vidaña, Eur. Phys. J. A 50 (2014).

[6] C. Fuchs and H.H. Wolter, Eur. Phys. J. A 30 (2006) 5.

[7] B. A. Brown, Phys. Rev. Lett. 111 (2013) 232502.

[8] Z. Zhang and L. W. Chen, Phys. Lett. B 726 (2013) 234.

[9] M. B. Tsang, Y. Zhang, P. Danielewicz, M. Famiano, Z. Li, W. G. Lynch, A. W. Steiner, Phys. Rev. Lett. 102 (2009) 122701.

[10] P. Danielewicz and J. Lee, Nucl. Phys. A 922 (2014) 1.

[11] C. J. Horowitz, E. F. Brown, Y. Kim, W. G. Lynch, R. Michaels, A. Ono, J. Piekarewicz, M. B. Tsang, H. H. Wolter, J. Phys. G 41 (2014) 093001.

[12] X. Roca-Maza, M. Centelles, X. Viñas, M. Warda, Phys. Rev. Lett. 106 (2011) 252501.

[13] S. Abrahamyan et al., Phys. Rev. Lett. 108 (2012) 112502.

[14] K. Kumar, PoS Bormio2012 (2012) 42.

[15] Jun Xu, Lie-Wen Chen, Che Ming Ko, Bao-An Li, Yu-Gang Ma, Phys. Rev. C 87 (2013) 067601.

[16] P. Danielewicz, R. Lacey, W.G. Lynch, Science 298 (2002) 1592.

[17] Bao-An Li, Phys. Rev. Lett. 88 (2002) 192701.

[18] P. Russotto et al., Phys. Lett. B 697 (2011) 471.

[19] Y. Leifels et al., Phys. Rev. Lett. 71 (1993) 963.

[20] D. Lambrecht et al., Z. Phys. A 350 (1994) 115.

[21] Qingfeng Li, Zhuxia Li, S. Soff, R. K. Gupta, M. Bleicher, H. Stöcker, J. Phys. G 31 (2005) 1359.

[22] Q. Li, Z. Li, S. Soff, M. Bleicher, H. Stöcker, J. Phys. G 32 (2006) 151.

[23] Q. Li, Z. Li, S. Soff, M. Bleicher, H. Stöcker, J. Phys. G 32 (2006) 407.

[24] M.D. Cozma, Phys. Lett. B 700 (2011) 139.

[25] W. Trautmann and H.H. Wolter, Int. J. Mod. Phys. E 21 (2012) 1230003.

[26] W. Trautmann, PoS Bormio2014 (2014) 036.

[27] P. Russotto et al., Eur. Phys. J. A 50 (2014) 38.

[28] P. Russotto et al., Phys. Rev. C 94 (2016) 034608.

[29] P. Russotto et al., EPJ WebConf. 88 (2015) 00022.

[30] Th. Blaich et al., Nucl. Instrum. Methods Phys. Res. A 314 (1992) 136.

[31] J. Łukasik et al., Nucl. Instrum. Methods Phys. Res. A 709 (2013) 120.

[32] A. Schüttauf et al., Nucl. Phys. A 607 (1996) 457. 
[33] A. Pagano et al., Nucl. Phys. A 734 (2004) 504.

[34] E. De Filippo and A. Pagano, Eur. Phys. J. A 50 (2014) 32.

[35] D.G. Sarantites et al., Nucl. Instr. and Meth. A 381 (1996) 418.

[36] J.-Y. Ollitrault, preprint nucl-ex/9711003 (1997).

[37] A. Andronic, J. Łukasik, W. Reisdorf, W. Trautmann, Eur. Phys. J. A 30 (2006) 31.

[38] S. A. Bass et al., Progr. Part. Nucl. Phys. 41 (1998) 255.

[39] Q. Li and M. Bleicher, J. Phys. G 36 (2009) 015111.

[40] Qingfeng Li, C. Shen, C. Guo, Y. Wang, Z. Li, J. Łukasik, W. Trautmann, Phys. Rev. C 83 (2011) 044617.

[41] Y. Wang, C. Guo, Q. Li, H. Zhang, Z. Li, W. Trautmann, Phys. Rev. C 89 (2014) 034606.

[42] M.D. Cozma, Y. Leifels, W. Trautmann, Q. Li, P. Russotto, Phys. Rev. C 88 (2013) 044912.

[43] C. B. Das, S. D. Gupta, C. Gale, B.-A. Li, Phys. Rev. C 67 (2003) 034611.

[44] A. Le Fèvre, Y. Leifels, W. Reisdorf, J. Aichelin, and Ch. Hartnack, Nucl. Phys. A 945 (2016) 112.

[45] W. Reisdorf et al., Nucl. Phys. A 781 (2007) 459.

[46] Jun Hong and P. Danielewicz, Phys. Rev. C 90 (2014) 024605.

[47] Zhi-Gang Xiao, Gao-Chan Yong, Lie-Wen Chen, Bao-An Li, Ming Chang, Guo-Qing Xiao, Nu Xu, Eur. Phys. J. A $\mathbf{5 0}$ (2014) 37.

[48] M. D. Cozma, Phys. Lett. B 753 (2016) 166.

[49] A. W. Steiner, J. M. Lattimer, and E. F. Brown, Astrophys. J. Lett. 765 (2013) L5.

[50] R. Subedi et al., Science 320 (2008) 1476.

[51] O. Hen et al., Science 346 (2014) 614.

[52] Wen-Mei Guo, Gao-Chan Yong, Yongjia Wang, Qingfeng Li, Hongfei Zhang, and Wei Zuo, Phys. Lett. B 738 (2014) 397.

[53] NeuLAND Technical Design Report, submitted to FAIR (2011). 